\title{
HACIA UNA BIBLIOTECA ELECTRÓNICA TEXTUAL DEL TEATRO EN ESPAÑOL DE 1868-1936 (BETTE)
}

\author{
José Calvo Tello* \\ Sila Gómez ${ }^{* *}$ \\ Juana María González ${ }^{* \star *}$ \\ Rocío Vilches $^{* * * *}$
}

RESUMEN: En este artículo se presenta el proyecto BETTE que consiste en la creación de una biblioteca electrónica textual del teatro en español de 1868-1936. Este proyecto lo lleva a cabo el grupo GHEDI (Grupo de Humanidades y edición digital) de la Universidad Internacional de la Rioja (UNIR). El texto presenta un breve panorama de los cambios introducidos por el impacto de lo digital en el ámbito de la edición textual y ofrece un estado de la cuestión de los proyectos que actualmente desarrollan las bibliotecas electrónicas que utilizan XML-TEI para codificar textos electrónicos. Asimismo, en él se presta atención a los llamados lenguajes de marcado y se presentan algunas características de TEI (Text Encoding Initiative). Finalmente, se describen las particularidades de nuestro proyecto, objetivos y primeros avances.

PALABRAS CLAVE: Biblioteca electrónica textual. Teatro español. Edición electrónica. Lenguajes de marcado. TEI (Text Encoding Initiative).

\section{INTRODUCCIÓN}

Parece obligado en la presentación de un proyecto sobre la creación de una biblioteca electrónica textual de teatro en España dedicar unas pocas líneas a contextualizar el origen y alcance de lo virtual frente al concepto tradicional de biblioteca dentro de los estudios literarios.

\footnotetext{
*Universität Würzburg, Würzburg, Alemanha. E-mail: jose.calvo@uni-wuerzburg.de

** Universidad Internacional de La Rioja, España. E-mail: sila.gomez@unir.net

*** Universidad Internacional de La Rioja, España. E-mail: juanamaria.gonzalez@unir.net

*** Universidad Internacional de La Rioja, España. E-mail: rocio.vilches@unir.net
}

\section{(cc) BY-NC-ND}

Esta obra está licenciada com uma licença Creative Commons. 
Decir que el paso de la era Gutenberg a la era digital ha supuesto una revolución que ha influido en todos los ámbitos de nuestra cotidianeidad es una afirmación que, por obvia, no es menos radical en sus presupuestos.

Esta transformación ha sido igualmente relevante en el campo de los estudios literarios. En un primer momento, el concepto de "hipertexto" se impuso como metáfora que condensaba la trascendencia de estos cambios. El hecho de que un texto pudiera entrar en relación, mediante hipervínculos, con otros textos u otros lugares del mismo, rompía con una linealidad que se había visto como elemento configurador de la escritura clásica y venía a sancionar en la práctica lo que algunos teóricos de la literatura como Barthes o Derrida habían postulado en sus estudios de literatura posmoderna: se declaró, así pues, la "muerte" del autor y el nacimiento de un nuevo tipo de lector, que con su activo papel se convertía en coautor del texto literario (Landow, 2008).

La multilinealidad se erigía, por tanto, en una característica esencial del hipertexto. Además de esta, el hipertexto traía emparejado otra serie de cualidades que favorecían esta nueva visión: como señala Pajares (2004, p. 2122) el carácter multimedia, el formato múltiple, la interactividad, la dinamicidad y la conexión mundial seguían sumando novedades a esta nueva era digital, de las que seguimos extrayendo ventajas.

El paso del tiempo y la definitiva implantación de la tecnología informática en nuestro día a día ha supuesto, por fin, la atenuación de algunos de estos excesos de la crítica. Pero, al mismo tiempo, ha traído la consolidación de una serie de herramientas y métodos de trabajo, distintos o complementarios a la función del hipervínculo, que ofrecen una valiosísima ayuda a los estudios filológicos.

Las ediciones y las bibliotecas electrónicas son una prueba incontestable de este nuevo universo que se ofrece al investigador literario. Con nuestro proyecto de creación de una biblioteca electrónica textual del teatro en español (1868-1936) pretendemos sumarnos a esta nueva manera de acceder a los textos, ofreciendo a los investigadores un instrumento que les permita acceder a un corpus de obras Texto Digital, Florianópolis, Santa Catarina, Brasil, v. 11, n. 2, p. 171-184, jul./dez. 2015. ISSNe: 1807-9288. 
teatrales, así como profundizar en su estudio ayudados por las enormes potencialidades que trae aparejada la era digital.

\section{LAS BIBLIOTECAS ELECTRÓNICAS}

El desarrollo de las bibliotecas electrónicas en los últimos años hace necesario un balance de los progresos en este ámbito y pone de manifiesto la necesidad de superar el modelo de biblioteca basado en la acumulación de textos para convertirlo en un espacio de conocimiento (Lucía Megías, 2009).

En un primer nivel, las bibliotecas virtuales facsimilares ofrecen tanto facsímiles de obras antiguas como estudios o ediciones en formato PDF. Sin embargo, este tipo de bibliotecas prima la accesibilidad frente a la recuperabilidad, esto es, facilita el acceso a los textos pero no incorpora de forma generalizada motores de búsqueda.

Por su parte, las bibliotecas virtuales especulares ponen a disposición del usuario textos digitalizados de ediciones existentes y transcripciones. Este tipo de bibliotecas permite las búsquedas e incorpora concordancias, pero también tiene deficiencias. En efecto, las bibliotecas que codifican los textos en TXT dada su compatibilidad con cualquier sistema operativo suelen ofrecer escasa calidad textual en las transcripciones digitalizadas. Respecto a la digitalización de ediciones analógicas, los resultados son desiguales, pues si bien en algunas ocasiones no se indican las ediciones que han servido de base o no se justifica su elección, lo cierto es que existen bibliotecas donde prima el rigor filológico.

Una tercera posibilidad es la que ofrecen las bibliotecas cuyos textos han sido editados expresamente para la divulgación en la red, donde también podemos encontrar trabajos de mayor y menor calidad.

Frente a estos modelos que utilizan las ventajas de la informática mayoritariamente con un afán de almacenamiento, surgen nuevas posibilidades de edición hipertextual. En este sentido, resultan destacables los modelos de Texto Digital, Florianópolis, Santa Catarina, Brasil, v. 11, n. 2, p. 171-184, jul./dez. 2015. ISSNe: 1807-9288. 
edición sinóptica integral o genética que priman la personalización y la interactividad para ofrecerle al usuario unos materiales que pueda utilizar según sus necesidades. Así, en palabras de Lucía Megías (2009), se supera el modelo del "incunable del manuscrito" y se consolidan nuevos modelos hipertextuales.

Las bibliotecas virtuales hipertextuales se diferencian de las anteriores porque presentan de forma simultánea materiales de distinta naturaleza, incorporan herramientas de apoyo y diseñan nuevos modelos editoriales hipertextuales.

Otro tipo de clasificación de las bibliotecas digitales es la que propone Lucía Megías (2010) de acuerdo con un criterio de finalidad. Según esta ordenación del universo textual de las bibliotecas digitales, es necesario señalar la existencia de dos tipologías: las bibliotecas virtuales y las bibliotecas textuales. En virtud de esta distinción, las primeras clasifican el material bibliográfico de una determinada institución y priman el objeto, mientras que las segundas, en las que se enmarca el proyecto que presentamos, sitúan el texto, el contenido, en el centro de sus intereses.

En cuanto a su desarrollo tecnológico, las bibliotecas digitales han incorporado paulatinamente los avances de la informática y, dada la amplitud de formatos con los que cuenta la edición electrónica en la actualidad, han priorizado la adopción de estándares ampliamente aceptados que hagan posible el desarrollo futuro de los textos.

Nuestro proyecto de creación de una biblioteca electrónica textual del teatro en español de 1868-1936 (BETTE) ha partido de un análisis de las prestaciones que nos ofrecen los distintos formatos existentes y ha optado por realizar el marcado en XML-TEI. Este lenguaje de marcado es el estándar actual para marcar los textos de manera semántica. Decenas de grupos académicos lo utilizan para 
codificar textos con fines de lingüística de corpus, diccionarios, manuscritos, textos literarios, correspondencia personal, etcétera ${ }^{1}$.

Algunos ejemplos de importantes proyectos que en la actualidad codifican y publican su código en XML-TEI son:

- En el ámbito angloparlante podemos señalar el University of Oxford Text Archive, un portal que contiene más de 5000 textos codificados en TEI y otros muchos textos en otros formatos. Otro proyecto interesante de menor extensión pero de mayor detalle es el Shakespeare's Plays from Folger Digital Texts, dirigido por Mowat y Werstine.

- En el ámbito alemán podemos señalar como proyectos generalistas el proyecto TextGrid, actualmente dirigido por Wolfram Horstmann, en el que cooperan diez instituciones académicas alemanas y que contiene textos de más de 1200 autores; y el Deutsches Text Archiv, un corpus textual de referencia en alemán que cubre desde 1600 hast 1900 y que en la actualidad ha publicado más de 1600 obras.

- En el ámbito francés, el proyecto de Théâtre Classique, dirigido por Paul Fièvre, ofrece más de 700 piezas de teatro francés escritas entre los siglos XVII y XVIII.

En el ámbito español se observan algunas iniciativas importantes, aunque por ahora no hay proyectos análogos que cubran la mayor parte del canon literario en español y que publiquen en XML-TEl. Algunos de los proyectos de ámbito hispánico que actualmente publican su código XML-TEI son:

- El Proyecto Tesoro de la Universidad Carlos III de Madrid, centrado en el teatro del Siglo de Oro y que hasta la fecha ha publicado 5 textos.

- El proyecto ReMetCa, dirigido por González-Blanco, un repertorio métrico digital de la poesía medieval castellana que codifica y publica los ejemplos en TEl.

1 Véase la web de "Special Interest Groups" en la web de TEl http://www.tei-c.org/Activities/SIG/. Acceso: 20 de abril de 2015.

Texto Digital, Florianópolis, Santa Catarina, Brasil, v. 11, n. 2, p. 171-184, jul./dez. 2015. ISSNe: 1807-9288. 
- La Biblioteca Digital del Pensamiento Novohispano, que publica ediciones críticas de textos del siglo XVII en Nueva España.

Dentro del ámbito hispánico hay otro conjunto de proyectos que trabajan con XML-TEI o con otros lenguajes de marcado, pero que no publican sus resultados en este lenguaje. Entre ellos:

- El proyecto Teatro Español del Siglo de Oro, que desde 1997 ofrece licencias para acceder a 800 piezas de teatro español escritas en el Siglo de Oro.

- La Association for Hispanic Classical Theater, que ha publicado en su web alrededor de 250 obras, la mayoría en formato HTML.

- El proyecto Arte Lope, de la Universitat de València, dirigido por Joan Oleza Simó, que ofrece más de cien obras de Lope en HTML sobre las que se pueden visualizar mediante CSS algunos aspectos semántico-textuales.

- Clásicos Hispánicos es una colección de libros electrónicos en formato ePUB (es decir XHTML) y mobi, dirigida por Pablo Jauralde, que publica ediciones cuidadas filológicamente preparadas por especialistas en el área.

- Por último la Biblioteca Virtual Miguel de Cervantes es un ambicioso proyecto que señala que codifica parte de sus textos en TEl. Lamentablemente no lo publica, sino que genera archivos HTML no validados y repletos de malas prácticas ${ }^{2}$. Este portal no solo publica textos en lenguajes de marcado, también contiene ediciones facsímiles en archivos de imágenes, archivos PDFs, enlaces a fuentes externas, etcétera. Nos es difícil cuantificar cuántos textos literarios han sido publicados en este portal en lenguaje de marcado, desde luego varios cientos, probablemente miles ${ }^{3}$.

\footnotetext{
2 En su código HTML se encuentran definición de estilos tanto en HTML como en hojas CSS, saltos de líneas $(<b r />)$, <span> vacíos o sin definir, elementos de párrafo vacíos para crear espacio, uso de estilos sin nombres semánticos, uso de elementos obsoletos (considerados deprecated) como <big>, <small>, <sup>, <sub> y un largo etcétera.

${ }^{3}$ El portal no aporta ese dato ni han sido capaces de darnos el dato en comunicación personal.
} 
A continuación explicaremos algunas características de los llamados lenguajes de marcado, concretamente en TEI (Text Encoding Initiative) para señalar sus características y ventajas, así como para justificar su uso en nuestro proyecto.

\section{LENGUAJES DE MARCADO. TEXT ENCODING INITIATIVE (TEI) ${ }^{4}$}

Uno de los grandes avances en el ámbito de la edición electrónica ha sido la incorporación de las tecnologías de marcado textual (Markup Technologies). Huitfeldt (2014) indica que el término "marcado" hace referencia al uso de una serie de etiquetas en un texto electrónico que nos permiten resaltar determinados elementos del mismo; esto es, seleccionado un elemento del texto, aplicarle un código que nos permita identificarlo en función de unas categorías predefinidas (por ejemplo: autor, persona, lugar, etc.). Aplicar este tipo de técnicas permite aproximarnos a los textos de muy distintas maneras "for search and retrieval, computer-assisted concordancing, collation, word-frequency counts, collocation analysis, linguistic or stylistic analysis" (Huitfeldt, 2014) y así avanzar exponencialmente en su estudio.

Un ejemplo muy sencillo sería codificar los títulos literarios que aparecen en la correspondencia cruzada entre dos escritores y ponerlos en relación con su obra creativa. Esto podría permitir a los investigadores identificar qué libros influyeron más en su obra y estilo o determinar si estos compartían los mismos intereses literarios.

Por otra parte, Huitfeldt también afirma que adherirse a los estándares de marcado internacional es una necesidad y una garantía para la supervivencia en el tiempo del texto que se edita electrónicamente así como para favorecer su interoperabilidad y accesibilidad en distintos formatos, softwares o sistemas.

\footnotetext{
${ }^{4}$ Para abordar este apartado hemos tenido en cuenta el trabajo de James Cummings "The Text Encoding Initiative and the study of literature" incluido en la ya clásica obra, coordinada por Ray Siemens y Susan Schreibman, A Companion to Digital Literary Studies (2008) y el artículo de la propia Susan Schreibman "Digital Scholarly Editing" en Literary Studies in the Digital Age. An Evolving Anthology (2013).
}

Texto Digital, Florianópolis, Santa Catarina, Brasil, v. 11, n. 2, p. 171-184, jul./dez. 2015. ISSNe: 1807-9288. 
En este apartado vamos a centrarnos en Text Encoding Initiative (TEI) que es un consorcio internacional que mantiene la TEI Guidelines (Guía TEI), que presenta uno de los lenguajes de marcado más extendidos entre la comunidad académica.

La Text Encoding Initiative (TEI) surgió de la necesidad de creación de unos estándares internacionales de marcado textual que permitieran a los distintos grupos de investigación que trabajan con textos electrónicos colaborar entre sí. El origen de esta iniciativa debe circunscribirse a una conferencia celebrada en Vassar College (Poughkeepsie) en noviembre en 1987 donde se expusieron estas preocupaciones y distintas propuestas por parte de diversos grupos de investigación. Los patrocinadores iniciales del proyecto TEl fueron The Association of Computers in the Humanities $(\mathrm{ACH})$, The Association for Computational Linguistics (ACL) y The Association of Literary and Linguistic Computing (ALLC). TEl es mantenido ahora como consorcio internacional de estas instituciones así como de miembros institucionales y suscriptores individuales.

El objetivo general del proyecto TEl es mantener unos estándares de lenguaje de marcado (desde 2002 en la versión P4 basado en XML) para la codificación y uso de textos electrónicos, mayoritariamente de las disciplinas relacionadas con la Lingüística y la Literatura. El proyecto ofrece esquemas, herramientas para la creación de propios esquemas (Roma), documentación en forma de guías, ejemplos de proyectos, hojas de estilos y de conversión, etcétera. El consorcio está compuesto por comités especializados en documentación textual, representación textual, interpretación y análisis textual. Estos comités, en la medida de lo posible, tratan de hacer compatible el sistema TEI con los restantes sistemas de marcado ya existentes.

La repercusión del TEl ha sido altísima, hasta el punto de que ha influenciado el desarrollo de numerosos estándares web (Cummings, 2008). El formato está cada vez más extendido y es el recomendado internacionalmente para garantizar la preservación y posibilidad de intercambio (interoperabilidad) de las fuentes 
textuales de tipo electrónico para proyectos y trabajos en el ámbito de las humanidades.

Uno de los aspectos más reseñables de la guía TEI es que está en continua revisión. Las propuestas de actualización de TEI se revisan de forma continua y en la actualidad han alcanzado su quinta versión principal (P5). Las propuestas abarcan un amplio rango de temas de interés para la comunidad TEI y también suponen una historia viva de las preocupaciones del momento en el campo de las Humanidades Digitales.

La creciente popularidad de TEI conlleva el reto de adaptar una guía general a proyectos individuales de toda índole, cada uno con necesidades muy específicas. Adicionalmente, como TEl está concebido como un sistema general, contiene mucho más de lo que cualquier proyecto individual puede necesitar. Este doble reto puede dar lugar a una mayor divergencia y fragmentación, lo cual sería precisamente lo opuesto a los objetivos iniciales de TEI. Sin embargo, este riesgo de fragmentación se antoja como necesario, dadas las ventajas asociadas a permitir cierta personalización y adaptación del estándar TEl a cada proyecto concreto.

Debido a esta dicotomía entre estandarización y especialización, la creación de guías y estándares digitales es una tarea en constante evolución. Queda mucho por hacer y los esfuerzos de organizaciones como TEI son encomiables para crear un estándar común de acuerdo con el cual se puedan desarrollar aplicaciones y entornos de investigación cada vez más potentes.

A continuación pasaremos a presentar nuestro proyecto de creación de una biblioteca textual del teatro en español, sus objetivos, particularidades y primeros avances, así como las características del código que vamos a utilizar.

\section{HACIA UNA BIBLIOTECA ELECTRÓNICA TEXTUAL DEL TEATRO EN ESPAÑOL DE 1868-1936 (BETTE)}


Como ya hemos apuntado al comienzo de este trabajo, el Grupo de Humanidades y Edición Digital (GHEDI) de la UNIR centra actualmente sus esfuerzos en la creación de una biblioteca electrónica textual del teatro español publicado entre 1868 y 1936. El plan de trabajo para el año 2015 contempla la edición de cinco obras teatrales: Doña Perfecta (1896) y Casandra (1910), de Benito Pérez Galdós, Mancha que limpia (1895) y A fuerza de arrastrarse (1905), de José Echegaray, y Juan José (1895), de Joaquín Dicenta.

Respecto al tipo de edición, codificaremos los textos en XML-TEI. Concretamente, nuestra labor consistirá en marcar los diferentes tipos de fragmentos del texto de manera descriptiva. Gracias a la codificación utilizada este trabajo pondrá a disposición de la comunidad investigadora textos que separen de manera coherente aquellas principales secciones de una obra de teatro: paratextos en general (listado de personajes, introducción, acotaciones, descripciones de escena, nombres de personajes) y texto literario. De esta manera se superará el modelo de edición en HTML que ofrecen, entre otros, proyectos como el de la Biblioteca Virtual Cervantes y que ofrecen resultados tan pobres para la investigación.

En nuestra página web ofreceremos también la posibilidad de lectura del archivo mediante hojas de estilo CSS, aunque nuestro objetivo primordial no es ofrecer textos para realizar una lectura personal y cuidada, es decir close reading (Moretti, 2005, p. 1). Asimismo, y gracias a las características de este tipo de edición por el que optamos, no solo obtendremos un primer corpus textual sino que este nos proporcionará la base para sucesivos proyectos más concretos. En este sentido, la publicación de estos textos como eBooks o apps o la realización de ediciones facsímiles o dirigidas a un público determinado son solo algunas de las líneas de trabajo que posibilita la formación de este corpus textual inicial.

En la actualidad el proyecto se encuentra en fase de pruebas y construcción. Un primer esquema ha sido constituido mediante la herramienta Roma y contiene alrededor de 50 etiquetas. El esquema está basado en la versión P5 de XML-TEI y ha sido basado en la versión LITE, reduciéndose notablemente los elementos y Texto Digital, Florianópolis, Santa Catarina, Brasil, v. 11, n. 2, p. 171-184, jul./dez. 2015. ISSNe: 1807-9288. 
atributos posibles. Publicaremos el esquema como parte de nuestro corpus para que terceros investigadores puedan trabajar con los textos.

Con este esquema ya hemos codificado la obra de Galdós Electra. Durante los siguientes meses esperamos definir un flujo de trabajo concreto, así como introducir posibles modificaciones del esquema para adaptarlo tanto a las peculiaridades históricas y culturales de los textos, como a nuestros objetivos como proyecto.

\title{
TOWARDS A TEXTUAL ELECTRONIC LIBRARY OF THEATHER IN SPANISH FROM 1868 TO 1936 (BETTE)
}

\begin{abstract}
This article makes an introduction to the BETTE project, which consists in the creation of an electronic textual library of the Spanish language theater during 1868-1936. The BETTE project is being done by GHEDI (Humanities and Digital Edition Group) of the Universidad Internacional de la Rioja (UNIR). The article gives a brief overview of the changes being driven by digitalization in the field of textual edition, and provides a state of the matter regarding the projects currently being developed by the electronic libraries which use XML-TEI to encode electronic text. Additional focus is made on the markup languages and on some of the characteristics of TEI (Text Encoding Initiative). Finally, some of the particularities, objectives and initial progress of BETTE are presented.
\end{abstract}

KEYWORDS: Electronic textual library. Spanish Theater. Electronic editing. Markup languages. TEl (Text Encoding Initiative).

\section{REFERENCIAS BIBLIOGRÁFICAS}

CARIDAD SEBASTIÁN, Mercedes. Proyecto TESORO. Madrid: Universidad

Carlos III. Disponible en:

<http://www.bib.uc3m.es/ nogales/xml/tesoro/index.html>. Acceso: 20 de abril de 2015. 
CUMMINGS, James. The Text Encoding Initiative and the Study of Literature. En SIEMENS, Ray; SCHREIBMAN, Susan (eds.). A Companion to Digital Literary Studies. Oxford: Backwell, 2008. Disponible en: <http://www.digitalhumanities.org/companionDLS/\%29>. Acceso: 20 de abril de 2015.

FIĖVRE, Paul. Théâtre Classique. Paris: 2009. Disponible en: <http://theatreclassique.fr/>. Acceso: 20 de abril de 2015.

GONZÁLEZ-BLANCO, Elena (dir). ReMetCa. Madrid: UNED, 2011. Disponible en: <www.remetca.uned.es>. Acceso: 20 de abril de 2015.

HOSTMANN, Wolfram (dir). TextGrid. Göttingen: Georg-August-Universität Göttingen, 2006. Disponible en: < https://www.textgrid.de/>. Acceso: 20 de abril de 2015.

HUITFELDT, Claus. Markup technology and textual scholarship. En APOLON, Daniel; BÉLISLE, Claire; RÉGNIER, Philippe. Digital Critical Editions. Exploring the interweaving of traditional and digital textual scholarship. Illinois: Illinois University Press, 2014 (Kindle edition).

JAURALDE POU, Pablo (dir.). Clásicos Hispánicos. Stein bei Nürnberg: More Than Books, 2012. Disponible en: <http://www.clasicoshispanicos.com/>. Acceso: 20 de abril de 2015.

KLEIN, Wolfgang (dir.). Deutsches Text Archiv. Berlin: Brandenburgische Akademie der Wissenschaften, 2007. Disponible en: <http://deutschestextarchiv.de/>. Acceso: 20 de abril de 2015.

LANDOW, George P. Hipertexto 3.0. Teoría crítica y nuevos medios en la era de la globalización. Barcelona: Paidós, 2006. 
LUCÍA MEGÍAS, José Manuel. De las bibliotecas digitales a las plataformas de conocimiento (notas sobre el futuro del texto en la era digital). En ARBOR ALDEA, Mariña; GUIADANES, Antonio F. (eds.). Estudos de edición crítica e lírica galegoportuguesa. Verba. Anuario galego de Filoloxía. Anexo 67. Santiago de Compostela: Universidade de Santiago de Compostela, 2010, p. 369-401.

LUCÍA MEGÍAS, José Manuel. La edición crítica hipertextual. Hacia la superación del incunable del hipertexto. En CASTILLO MARTíNEZ, Cristina; RAMÍREZ LUENGO, José Luis (eds.). Lecturas y textos en el siglo XXI. Nuevos caminos en la edición textual. Lugo: Axac (Logophiles, 3), 2009, p. 11-74.

MORETTI, Franco. Graphs, Maps, Trees. London-New York: Verso, 2005.

MOWAT, Barbara; WERSTINE, Paul. Shakespeare's Plays from Folger Digital Texys. Washington: Folger, 2010. Disponible en:

<http://www.folgerdigitaltexts.org/>. Acceso: 20 de abril de 2015.

OLEZA SIMÓ, Joan (dir.). Biblioteca digital Artelope. Valencia: Universitat de València, 2013. Disponible en: <http://artelope.uv.es/biblioteca/>. Acceso: 20 de abril de 2015.

PAJARES TOSCA, Susana. Literatura Digital. El paradigma hipertextual. Cáceres: Universidad, 2004.

PRIANI SAISÓ, Ernesto (dir.). Biblioteca Digital del Pensamiento Novohispano. Ciudad de México: Universidad Nacional Autónoma de México, 2010. Disponible en: < http://www.bdpn.unam.mx/>. Acceso: 20 de abril de 2015.

SCHREIBMAN, Susan. Digital Scholarly Editing. En Literary Studies in the Digital Age. An Evolving Anthology (2013), in MLA Commons. Disponible en: <http://dlsanthology.commons.mla.org/>. Acceso: 20 de abril de 2015. 
SIMÓN PALMER, Ma del Carmen (dir.). Teatro español del Siglo de Oro. Ann Arbor, Michigan, USA: ProQuest, 1997. Disponible en:

<http://teso.chadwyck.co.uk/>. Acceso: 20 de abril de 2015.

TEl Guidelines (Guía TEI). 2015. Disponible en: <http://www.teic.org/Guidelines/>. Acceso: 20 de abril de 2015.

Text Encoding initiative (TEI). 2015. Disponible en: <http://www.tei-c.org>. Acceso: 20 de abril de 2015.

WYNNE, Martin (dir.). University of Oxford Text Archive. Oxford: University of Oxford, 1976. Disponible en: <http://ota.ox.ac.uk/>. Acceso: 20 de abril de 2015.

Texto recebido em: 19/10/2015.

Texto aceito em: 11/12/2015. 